
\title{
28 Research Square \\ Hydrolyzed FB1 (aminopentol) detection on urine of HPV positive Mexican women: A preliminary study
}

\section{M. Ángeles Ramírez-Cisneros ( $\square$ angelesrc@uaem.mx )}

Centro de Investigaciones Químicas, Universidad Autónoma del Estado de Morelos

\section{Vladimir Rivera}

Centro de Investigación en Dinámica Celular, Universidad Autónoma del Estado de Morelos

\section{Maria Yolanda Rios}

Centro de Investigaciones Químicas, Universidad Autónoma del Estado de Morelos

\section{Cruz Vargas-De-León}

Facultad de Matemáticas, Universidad Autónoma de Guerrero

\section{Sonia Dávila}

Centro de Investigación en Dinámica Celular, Universidad Autónoma del Estado de Morelos

\section{Raúl Peralta}

Centro de Investigación en Dinámica Celular, Universidad Autónoma del Estado de Morelos

\section{Research Article}

Keywords: HPV, Fumonisin B1, Hydrolyzed FB1 (aminopentol), Sphingolipids

Posted Date: March 8th, 2021

DOI: https://doi.org/10.21203/rs.3.rs-68390/v2

License: (c) (1) This work is licensed under a Creative Commons Attribution 4.0 International License.

Read Full License 


\section{Abstract}

Human Papillomavirus (HPV) infection is the most common sexually transmitted disease in humans. About $200 \mathrm{HPV}$ types have been described and classified into high and low oncogenic risk for its ability to transform the cell. Some main risk factors for HPV infection are multiple sexual partners, multiparity and immunosuppression. However, these factors can not completely explain the HPV prevalence in different world regions. Mexico has a high global burden, being important to determine other external biological factors implicated. The infection is mediated by surface receptors of basal stratum cells that bind to the viral capsid entering the cell by endocytosis. Some cell membrane components such as sphingolipids have a role in structure and membrane permeability. Besides, fumonisins are micotoxins structurally very similar to sphingolipids, fumonisin B1 (FB1) is the most abundant and most similar to some sphingolipids such ceramide. Fumonisins are produced by Fusarium fungus, widely distributed in maize, the main component of Mexican diet. Thus, it is possible that FB1 contamination be a factor to permeabilize the membrane expediting the HPV infection. In this preliminary study, we postulated that mechanism of HPV infection is mediated by contamination with FB1, hence, FB1 could be higher in women HPV positive. Urine samples from patients with HPV infection and healthy woman were analyzed by UPLC-MS, hydrolyzed FB1 was found only in the infected women, in contrast, in HPV negative women was not detected. Additional studies are needed to support the hypothesis of contamination with FB1 as biological risk factor for HPV infection.

\section{Introduction}

Persistent infection with high-risk human papillomavirus (HPV) is the main risk factor in the development of cervical cancer (CC). Near than 200 HPV types have been described in which at least 40 are sexually transmitted infecting the genital mucosa. Based on their oncogenic potential, these can be classified as oncogenic "high risk" and "low risk" [1]. HPVs are specific to the infection of cells of the basal stratum of squamous epithelial tissue. The introduction of virions into the cell is initiated by the interaction of the L1 protein with glycoproteins on the cell surface. It has also been associated with integrin alpha- 6 in the entry of the virus into the cell. Once it interacts with the cell, HPV enter the cell through clathrin-dependent endocytosis [2]. HPV infection is the most common sexually transmitted disease in the human population, but most HPV infections are cleared by the immune system within two years. The main risk factors for HPV infection are the early sexual initiation, multiple sexual partners, multiparity and immunosuppression [2]. Interestingly, HPV infection prevalence changes by geographical location. In North America, the prevalence is $13.8 \%$, South America 14.3\%, Europe, and Asia with $6.6 \%$ and $8.3 \%$ respectively while the regions with the highest prevalence are the African continent and Central America (including Mexico) with $22.9 \%$ and $20.5 \%$ respectively [3]. HPV infections are more frequent in developing countries, although the exposure to risk factors for HPV infection is similar between developed and developing regions [3]. 
On the other hand, a recent study determined that maize contaminated with a mycotoxin, fumonisin B1 (FB1), derived from a filamentous fungus is associated with the transmission of the human immunodeficiency virus (HIV) in Sub-Saharan Africa. The authors in this study hypothesize a relationship between the consumption of maize contaminated with FB1 and the increase in HIV transmission, suggesting that maize contaminated with FB1 is an important factor in the HIV transmission. According to the statistical model used, eliminating the "maize factor" from Sub-Saharan Africa could reduce HIV transmission by 58\% [4]. Other studies associate FB1 consumption with an increase in esophageal cancer and hepatocarcinoma, in regions of South Africa, India and China where they have a high exposure to this mycotoxin [5]. FB1 is the most toxicologically important and represents $70-80 \%$ of the total fumonisin found in nature, fumonisin B2 (FB2) represent 15 to $25 \%$ and fumonisin B3 (FB3) only represent $3-8 \%$. Fumonisin B contain a linear skeleton of 20 carbons, with a chemical structure similar to the long chain of sphingolipid precursors. The novo synthesis of sphingolipids begins with serine palmitoyl-CoA complex and also of the hydrolysis of complex sphingolipids. The main enzyme in this synthesis process is the ceramide synthetase (CS). This enzyme is blocked by FB1 through competitive inhibition and increases sphinganine and sphingosine (precursors of sphingolipids) favoring virus infection. Finally, produces a state of cellular permeability [6]. A detailed mechanism of interaction of FB1 with CS was described, briefly, the part of FB1 that has structural similarity to sphingoid bases may interact with the sphinganine binding site, while the negatively charged tricarboxylic acid groups may interact with the fatty acyl-CoA binding site [7]. However, when the tricarboxylic acid groups are separated from the FB1 by hydrolysis, the resulting product (aminopentol, AP1 or HFB1) not only acts as an inhibitor, but also as a substrate for the CS; aminopentol is acylated by CS to form N-palmitoyl-AP1. This supports the suggestion that the aminopentol part of FB1 occupies the sphinganine space in the enzyme. $\mathrm{N}$-palmitoyl-AP1 is an even more potent inhibitor of ceramide synthase and may therefore play a role in the toxicity of FB1 [7]. The decrease of complex sphingolipids and the accumulation of sphingoid bases (sphinganine and sphingosine) alter the cellular functions and alters the balance of cell death and replication and thus contributes to carcinogenesis, even more important is the effect on cellular permeability that would potentially facilitate interaction with pathogens, such as viruses [8].

On the other hand, the methods used for maize processing influence the final concentration of FB1. For example, the main form to obtain the mass of maize, nixtamalization, that consists in cooking maize grains in an alkaline solution of calcium hydroxide $\left(\mathrm{CaOH}_{2}\right)$, at a temperature close to the boiling point, reduces the concentration of FB1 in approximately $90 \%$ and the residual amount remains in its hydrolyzed form (HFB1), which is less potent in vitro than FB1 [9]. However, when the process is incomplete and the pericarp is not adequately eliminated, $31 \%$ of FB1 remains in its original form [9]. In Mexico, a study evaluated the consumption of tortillas (traditional food in Mexico, derived from maize) as a source of exposure to FB1 in humans. The consumption of maize was classified as high, medium, or low according to the consumption of tortillas. The urinary concentrations of FB1 were 3.5 times higher among women in the highest category of maize consumption compared to those in the lowest category. The authors concluded that the consumption of tortillas is directly related to the exposure of fumonisins [10]. The maize and its derivatives (such as tortilla) are essential foods in the Mexican diet, so the 
exposure to FB1 is greater than other countries. In this sense represents potentially a public health problem in Mexico. This biological factor has not yet been explored as a risk factor for HPV infection. In the present preliminary study, it is postulated that exposure to FB1 favors HPV infection. The objective of the present preliminary study is to determine levels of FB1 in urine of women positive and negative to HPV infection, to establish a possible association and propose a new risk factor in HPV infection.

\section{Materials And Methods}

\section{Biological samples and experimental design}

The clinical samples (urine and cervical scraping) were obtained from routine Papanicolaou (Pap) tests at the University Medical Center of the Autonomous University of the Morelos State (UAEM). All samples analyzed in the present study were obtained with informed consent and all experimental procedures were approved by the local ethics committee. During the collection of the biological samples, no additional or invasive procedures were performed on the patient. In this first experimental pilot stage, six samples analyzed were randomly taken from a batch of positive and negative HPV infections, 3 for each group. From every sample, the relevant clinical-epidemiological data were obtained, such as general data, exposure to risk factors for HPV infection, and frequency of consumption of maize and derivatives. To avoid bias in the result, FB1 levels (MARC author) and molecular detection of HPV (VR author) were performed by two different people in a blinded manner.

\section{DNA extraction}

The concentration of cells was performed by centrifugation. The cells contained in $1.5 \mu \mathrm{L}$ of the preservation buffer were centrifuged in a microcentrifuge at $5,000 \mathrm{~g}$ for 5 minutes. The supernatant was discarded. $300 \mu \mathrm{L}$ of nuclear lysis buffer $(100 \mathrm{mM} \mathrm{NaCl}, 10 \mathrm{mM}$ Tris $\mathrm{HCl}, 25 \mathrm{mM}$ EDTA and $0.5 \%$ SDS) and $30 \mu \mathrm{L}$ proteinase $\mathrm{K}$ were added and incubated in a thermal block at $56^{\circ} \mathrm{C}$ for $12 \mathrm{hrs}$. Next, $300 \mu \mathrm{L}$ of $5 \mathrm{M}$ ammonium acetate was added to precipitate the proteins and the tubes were left at $-20^{\circ} \mathrm{C}$ for 30 minutes. Subsequently, they were centrifuged at $5,000 \mathrm{~g}$ for 30 minutes. After, $600 \mu \mathrm{L}$ of isopropanol was added, incubated for $12 \mathrm{hrs}$ at $-20^{\circ} \mathrm{C}$ and centrifuged at $5,000 \mathrm{~g}$ for 15 minutes, the supernatant was decanted. The precipitate (DNA) was washed with ethanol and centrifugation at 3,000 g for 5 minutes. The procedure was repeated three times. Finally, the ethanol was decanted, and the DNA was dissolved with $30 \mu \mathrm{L}$ of nuclease-free water. The DNA was quantified in a NanoDrop 3300.

\section{Molecular detection and HPV genotyping}

Molecular detection and genotyping of HPV was performed using genomic microarrays, using the CLART ${ }^{8}$ HPV2 method (Clinical Array Technology Genomica). This system automatically detects $35 \mathrm{HPV}$ genotypes $(6,11,16,18,26,31,33,35,39,40,42,43,44,45,51,52,53,54,56,58,59,61,62,66,68,70$, $71,72,73,81,82,83,84,85$ and 89$)$. The system is based on the amplification of specific fragments of the viral genome and its subsequent hybridization with specific probes for each genotype of HPV printed on the microarray. The detection was carried out by amplifying a fragment of $450 \mathrm{bp}$ within the $\mathrm{L} 1$ region 
of the virus, highly conserved among the different types of HPV. However, this region has enough variations to differentiate each type of virus with specific probes. The detection system with CLART ${ }^{\circledR}$ human Papillomavirus 2 is based on the precipitation of an insoluble product in those areas of the microarray in which the hybridization of the amplified products with the specific probes takes place. During PCR, the amplified products are labeled with biotin. After amplification, these products are hybridized with their respective specific probes that are immobilized in specific and known areas of the microarray, followed by an incubation with streptavidin-peroxidase. For visualization, the PCR products are denatured at $95^{\circ} \mathrm{C}$ for 10 minutes and the procedure recommended by the manufacturer is followed. The preparation of the microarrays, the hybridization process, and the incubation were carried out following the manufacturer's recommendations. At the end of the process the plate was placed in the CAR to take the images of all the wells and be analyzed automatically. The processing of the data was done automatically.

\section{Detection of Fumonisins in urine}

\section{Samples pretreatment}

Urine samples were treated as previously reported [10] with minor modifications, urine was centrifuged for 10min, the supernatant was diluted 1:1 with distilled water. A standard solution of FB1 $(1 \mathrm{mg} / \mathrm{mL})$ was prepared and added to each sample as internal control $(0.1 \mathrm{ng} / \mathrm{mL})$. Samples were cleaned up into the $\mathrm{C}_{18}$ Oasis cartridges, MAX 3cc, as reported. Once the extraction solution (formic acid $2 \%$ in methanol) was individually obtained $(2 \mathrm{~mL})$, it was dried in a vacuum centrifuge and then reconstituted in $200 \mu \mathrm{L}$ of methanol / water in a 1:1 ratio. After the last step, the samples were placed in MS certificate vials. Positive and negative (control) samples were prepared by the same conditions.

\section{UPLC-MS-QTOF analysis}

The determination of FB's or hydrolyzed derivatives was performed using the ultrahigh resolution liquid chromatography (UPLC) technique coupled to a mass spectrometer quadrupole-time of flight (MS-QTOF) in an Infinity II Agilent equipment with a G6545A Agilent QTOF spectrometer with an ESI source. Injection volume was $8 \mu \mathrm{L}$ in a $\mathrm{C}_{18}$ column (SORBAX $2.7 \times 100 \mathrm{~mm}, 1.8 \mu \mathrm{M}$ ), a gradient elution at $25^{\circ} \mathrm{C}$ was carried out with a mobile phase at $0.3 \mathrm{~mL} / \mathrm{min}$ of $\mathrm{H}_{2} \mathrm{O}$ (solvent $\mathrm{A}$ ), and acetonitrile (solvent $\mathrm{B}$ ) both with formic acid $0.1 \%$ from 25 to $75 \%$ of solvent $B$ on $11 \mathrm{~min}$. Initial conditions were recovered before each injection. Mass conditions were, temperature for gas and sheath gas at 180 and $350{ }^{\circ} \mathrm{C}$ respectively, voltages for capillary and fragmentor were 3000 and $100 \mathrm{~V}$ respectively, an acquisition in positive mode from 400-900 $\mathrm{m} / \mathrm{z}$ was recorded. Positive, negative samples (control), and blank were analyzed by duplicate.

\section{Data analysis}

Data treatment was carried out on Profinder B.08 software using the extraction by formula for FB1 $\left(\mathrm{C}_{34} \mathrm{H}_{59} \mathrm{NO}_{15}\right)$ besides its hydrolyzed forms PHFB1 $\left(\mathrm{C}_{28} \mathrm{H}_{53} \mathrm{NO}_{10}\right)$ and HFB1 $\left(\mathrm{C}_{22} \mathrm{H}_{47} \mathrm{NO}_{5}\right)$, as well as FB2 or its isomer $\mathrm{FB} 3\left(\mathrm{C}_{34} \mathrm{H}_{59} \mathrm{NO}_{14}\right)$ and its hydrolyzed $\left(\mathrm{C}_{28} \mathrm{H}_{53} \mathrm{NO}_{9}, \mathrm{C}_{22} \mathrm{H}_{47} \mathrm{NO}_{4}\right)$, all aducts were allowed $(+\mathrm{H}$, 
$\left.+\mathrm{Na},+\mathrm{K},+\mathrm{NH}_{4}\right)$ and neutral loss of $\mathrm{H}_{2} \mathrm{O}$, compounds with more than 1000 counts were extracted, rt and $\mathrm{m} / \mathrm{z}$ tolerance were $0.15 \mathrm{~min}$ and of $10 \mathrm{ppm}$, respectively.

\section{Statistic analysis}

All of the analyses described below were carried out using R Statistical Software version 3.6.2. The onetailed Fisher's exact test was performed to the clinical-epidemiological variables and the FB1. The contingency tables with at least one structural zero are added 0.5 to each cell entry to measured the effect size with Cramer's V. We used the following function CramerV (Table2X2, conf.level=0.95, method $=$ c("fisheradj")) of R package DescTools (Signorell et mult. al., 2019). A Cramer's V between 0 and 0.3 is regarded as weak, one between 0.3 and 0.70 as medium, and greater than 0.7 is considered strong.

Subsequently, a principal component analysis (PCA) was analyzed using numerical variables (age and numbers of sexual partners) and dichotomy variables (vaccine and FB1) to determine possible clusters. Next, the scores of the first principal components were clustered using a K-means clustering. PCA and Kmeans clustering analyses were conducted using R package FactoMineR [11], factoextra [12] and ppclust [13].

\section{Results}

\section{Molecular detection of the HPV}

After the procedures of extraction of genetic material, detection and genotyping of HPV were performed, all the samples were complied with the internal controls of the HPV2 CLART system, two positive HPV belong to 82 type and one to 51 type were found (Table 1 ).

Table 1. HPV status.

\begin{tabular}{c|c|} 
Sample & HPV status \\
\hline RAA* & Positive (HPV 82 type) \\
\hline GBKX $^{*}$ & Positive (HPV 82 type) \\
\hline MZMJ* $^{*}$ & Positive (HPV 51 type) \\
\hline PII* & Negative \\
\hline CMIM* $^{*}$ & Negative \\
\hline VSB* & Negative \\
\hline \multicolumn{2}{|c|}{ Internal identification code for anonymity. }
\end{tabular}

\section{UPLC-QTOF analysis}

In this first blind pilot stage (preliminary study) all samples data were processed using the same conditions. HFB1 was detected on 3 of the 6 samples, after identification as positive and negative to HPV infection, HFB1 (aminopentol) was detected in all positive HPV samples in the order of $3.9 \times 10^{4}$ counts (it 3.9min, HRESIMS $m / z=406.3529$ for $[\mathrm{M}+\mathrm{H}]^{+}$), but any of the negative, while no FB1, PHFB1, FB2 or FB3 or its derivatives were detected in any sample included in this study (Figs 1-2). 
The clinical-epidemiological variables were not associated with HPV infection because the Cramer's V confidence intervals include zero. But, the effect size of the association between the HPV infection and FB1 is strong: Cramer's V $=0.75,95 \% \mathrm{Cl}[0.35,1.00]$ (Table 2). PCA clearly group samples according to HPV infection on positive and negative respectively with more than $83 \%$ of the variability explained by the two principal components, PC1 (56.4\%), PC2 (27.2\%) and PC3 (15.4\%) (Fig 3).

Table 2. HPV, clinical-epidemiological characteristics and FB1 association.

\begin{tabular}{|c|c|c|c|c|}
\hline Variables & HPV Positive & HPV Negative & P-value & $\begin{array}{c}\text { Cramer's V } \\
95 \% \text { CI }\end{array}$ \\
\hline Age & & & 0.20 & $0.52[0.00,0.90]^{*}$ \\
\hline$\leq 25$ & 3 & 1 & & \\
\hline$>25$ & 0 & 2 & & \\
\hline Years of schooling & & & 1.00 & $0.00[0.00,0.70]^{*}$ \\
\hline$\leq 12$ & 0 & 0 & & \\
\hline$>12$ & 3 & 3 & & \\
\hline Number of pregnancies & & & 0.50 & $0.33[0.00,0.91]$ \\
\hline$<1$ & 1 & 2 & & \\
\hline$\geq 1$ & 2 & 1 & & \\
\hline Numbers of sexual partners & & & 0.20 & $0.52[0.00,0.90]^{*}$ \\
\hline$\leq 1$ & 2 & 0 & & \\
\hline$>1$ & 1 & 3 & & \\
\hline Use of barrier contraceptives & & & 0.50 & $0.33[0.00,0.91]$ \\
\hline Yes & 2 & 1 & & \\
\hline No & 1 & 2 & & \\
\hline Alcohol consumption & & & 1.00 & $0.52[0.00,0.90]^{*}$ \\
\hline Yes & 1 & 3 & & \\
\hline No & 2 & 0 & & \\
\hline Age of onset of sexual activity & & & 1.00 & $0.52[0.00,0.90]^{*}$ \\
\hline$<18$ & 0 & 2 & & \\
\hline$\geq 18$ & 3 & 1 & & \\
\hline Tortilla consumption per day & & & 1.00 & $0.29[0.00,0.83]^{*}$ \\
\hline$\leq 1$ & 0 & 1 & & \\
\hline$>1$ & 3 & 2 & & \\
\hline HFB1 & & & 0.05 & $0.75[0.35,1.00]^{*}$ \\
\hline Yes & 3 & 0 & & \\
\hline No & 0 & 3 & & \\
\hline
\end{tabular}

* 0.5 is added to all cells

\section{PCA and Cluster Analysis}

A PCA was implemented to perform a dimensional reduction of four variables. Online Resource 1 shows that the five principal components explain the variance and that two principal components explain $83.4 \%$ of such variance. Online Resource 2 shows variable loading for principal component scores.

A K-means clustering algorithm was applied to the first two scores of principal components, in order to form clusters according their clinical-epidemiological characteristics and FB1. The statistical clustering analysis yielded two different clusters. Figure 3 shows that the two clusters coincide exactly with the HPV-positive (1) and HPV-negative groups (0). 


\section{Discussion}

The main risk factors for HPV infection have been widely described. These are factors related to sexual behavior such as the number of sexual partners and early sexual initiation. In general, infectious diseases are more frequent in developing regions, such is the case of HPV infection. However, this factor cannot fully explain the different prevalence of HPV infection in different regions of the world. The prevalence of HPV infection in Mexico, Central America and Africa are similar with about $20 \%$ of cases, while in North America, Europe and Asia, this prevalence is less than $10 \%$. The difference in prevalence is significant, although the exposure to risk factors is similar, that is, that the population in regions with lower prevalence have a similar sexual behavior $[14,15]$. In the present study, we postulate that other unexplored biological factors may be relevant in HPV infection. In the present preliminary study, we hypothesize that contamination by fumonisins in regions with high maize consumption, could be a risk factor in HPV infection. The acquisition of FB1 in the diet as a biological risk factor in HPV infection has not been described. Recently, the relationship between FB1 contamination and HIV infection is being explored. The authors conclude that contaminated maize that is used for human consumption is the source of exposure to FB1 [4]. This mycotoxin is cataloged by the International Agency for Research on Cancer in group 2B as possible carcinogens for humans, but, has not yet been linked to viral infections [16]. The presence of FB1 is documented in the Mexican population. In one study, the presence of FB1 in urine samples of Mexican women was evaluated, finding a relationship between the consumption of processed maize in the form of tortillas and the amount of FB1 detected in urine, the authors related the amount of tortilla consumption with the levels of FB1 in urine [10].

In the present study, we found a strong association between HPV infection (Cramer's V $=0.75$ ) and the presence of FB1 in its completely hydrolyzed form. Because the FB1 molecule can be hydrolyzed during maize processing, as well as possible degradation by human metabolism, for its detection a methodology based on UPLC-QTOF-MS was used, due to its high sensitivity and the possibility to detect fumonisins B or its hydrolyzed forms simultaneously. In this study FB1, FB2 or FB3 were not detected in any sample, even FB2 and FB3 hydrolyzed forms. However, FB1 in its completely hydrolyzed form (aminopentol, $\mathrm{C}_{34} \mathrm{H}_{59} \mathrm{NO}_{15}$ ) was detected only in urine from HPV positive women in order of $3.8 \times 10^{4}$ counts. Contamination of the cell with FB1 is postulated to lead to a state of cellular permeability through the inhibition of sphingolipide synthesis [17]. We postulate that FB1 could be facilitating HPV infection. Additional studies to demonstrate this mechanism are required. Although, the number of samples analyzed in the present study is relatively small, the statistical study shows a strong association between HPV infection and HFB1 levels.

\section{Conclusion}

In the present study, clearly the HPV infection have a strong association with FB1 through its completely hydrolyzed form known as aminopentol. Additional studies are necessary to support the hypothesis of contamination with FB1 as a biological risk factor for HPV infection. 


\section{Declarations}

\section{Funding}

RP thank PRODEP-SEP support. Chemical analyses were carried out at CIQ-LANEM, UAEM.

\section{Conflicts of interest}

The authors declare no conflict of interest.

\section{Ethics approval}

All experimental procedures were approved by the local ethics committee.

\section{Consent to participate}

All participants in the present study gave their informed consent.

\section{Consent for publication}

All participants in the present study gave their consent for publication.

\section{Availability of data and material}

Not applicable

\section{Code availability}

Not applicable

\section{Author Contributions}

MARC and MYR carried out the UPLC-MS analysis and processed the chemical data, VR performed the molecular detection of HPV, CVDeL, SD, RP conceived the complete study and wrote the article. All authors have read and contributed to the manuscript.

\section{Acknowledgments}

The authors thank Dr. Marco Capistrán and Sheila Jimenez for their contribution with the biological samples.

\section{References}

[1] Brianti P, De Flammineis E, Mercuri SR. Review of HPV-related diseases and cancers. New Microbiol. 2017;40(2):80-85. 
[2] Graham SV. The human papillomavirus replication cycle, and its links to cancer progression: a comprehensive review. Clin Sci (Lond) 2017; 131(17): 2201-2221. doi:10.1042/CS20160786.

[3] Torre LA, Islami F, Siegel RL, Ward EM, Jemal A. Global Cancer in Women: Burden and Trends. Cancer Epidemiol. Biomarkers Prev. 2017; 26(4): 444-457. doi:10.1158/1055-9965.EPI-16-0858.

[4] Williams JH, Grubb JA, Davis JW, et al. HIV and hepatocellular and esophageal carcinomas related to consumption of mycotoxin-prone foods in sub-Saharan Africa. Am J Clin Nutr. 2010; 92(1): 154-160. doi:10.3945/ajcn.2009.28761.

[5] Gelderblom WC, Abel S, Smuts CM, et al. Fumonisin-induced hepatocarcinogenesis: mechanisms related to cancer initiation and promotion. Environ Health Perspect. 2001; 109(2): 291-300. doi:10.1289/ehp.01109s2291.

[6] Stockmann-Juvala H, Savolainen K. A review of the toxic effects and mechanisms of action of fumonisin B1. Hum Exp Toxicol. 2008; 27(11):7 99-809. doi:10.1177/0960327108099525.

[7] Merrill AH Jr, Sullards MC, Wang E, Voss KA, Riley RT. Sphingolipid metabolism: roles in signal transduction and disruption by fumonisins. Environ Health Perspect. 2001; 109(2): 283-289. doi:10.1289/ehp.01109s2283.

[8] Marasas WF. Discovery and occurrence of the fumonisins: a historical perspective. Environ Health Perspect. 2001; 109(2): 239-243. doi:10.1289/ehp.01109s2239.

[9] Dombrink-Kurtzman MA, Dvorak TJ, Barron ME, Rooney LW. Effect of nixtamalization (Alkaline cooking) on fumonisin-contaminated corn for production of masa and tortillas. J Agric Food Chem. 2000; 48(11): 5781-5786. doi:10.1021/jf000529f.

[10] Gong YY, Torres-Sanchez L, Lopez-Carrillo L, et al. Association between tortilla consumption and human urinary fumonisin B1 levels in a Mexican population. Cancer Epidemiol Biomarkers Prev. 2008; 17(3): 688-694. doi:10.1158/1055-9965.EPI-07-2534.

[11] Le S, Josse J, Husson F. Facto Mine R: An R Package for Multivariate Analysis. J. Stat. Softw. 2008; 25(1): 1-18.

[12] Kassambara A, Mundt F. (2017). factoextra: Extractand Visualize the Results of Multivariate Data Analyses. R package version 1.0.5. https://CRAN.R-project.org/package=factoextra.

[13] Cebeci Z, Yildiz F, Kavlak AT, Cebeci C, Onder H. (2018). ppclust: Probabilistic and Possibilistic Cluster Analysis. R package version 0.1.1, URL https://CRAN.R-project.org/package=ppclust.

[14] Peralta-Rodríguez R, Romero-Morelos P, Villegas-Ruíz V, et al. Prevalence of human papillomavirus in the cervical epithelium of Mexican women: meta-analysis. Infect Agent Cancer. 2012; 7(1): 34. doi:10.1186/1750-9378-7-34. 
[15] Ribeiro AA, Costa MC, Alves RR, et al. HPV infection and cervical neoplasia: associated risk factors. Infect Agent Cancer. 2015; 10(16). doi:10.1186/s13027-015-0011-3.

[16] GLOBOCAN 2008 (IARC) Section of cancer information. http://globocan.iarc.fr.

[17] Riedel S, Abel S, Burger HM, van der Westhuizen L, Swanevelder S, Gelderblom WC. Differential modulation of the lipid metabolism as a model for cellular resistance to fumonisin B1-induced cytotoxic effects in vitro. Prostaglandins Leukot. Essent. Fatty Acids. 2016; 109: 39-51.

doi:10.1016/j.plefa.2016.04.006.

\section{Figures}

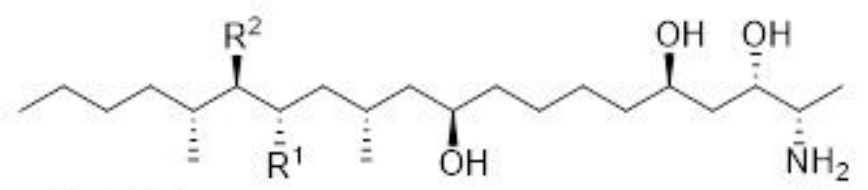

FB1 $\quad R^{1}=R^{2}=T C A$

PHFB1 $R^{1}, R^{2}=T C A, O H$

HFB1 $R^{1}=R^{2}=O H$

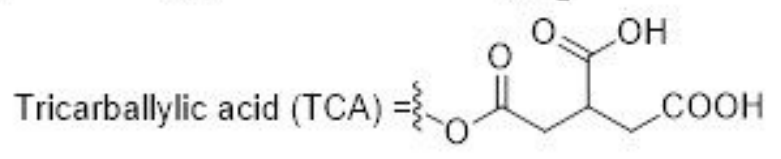

\section{Figure 1}

FB1 and its hydrolyzed forms 


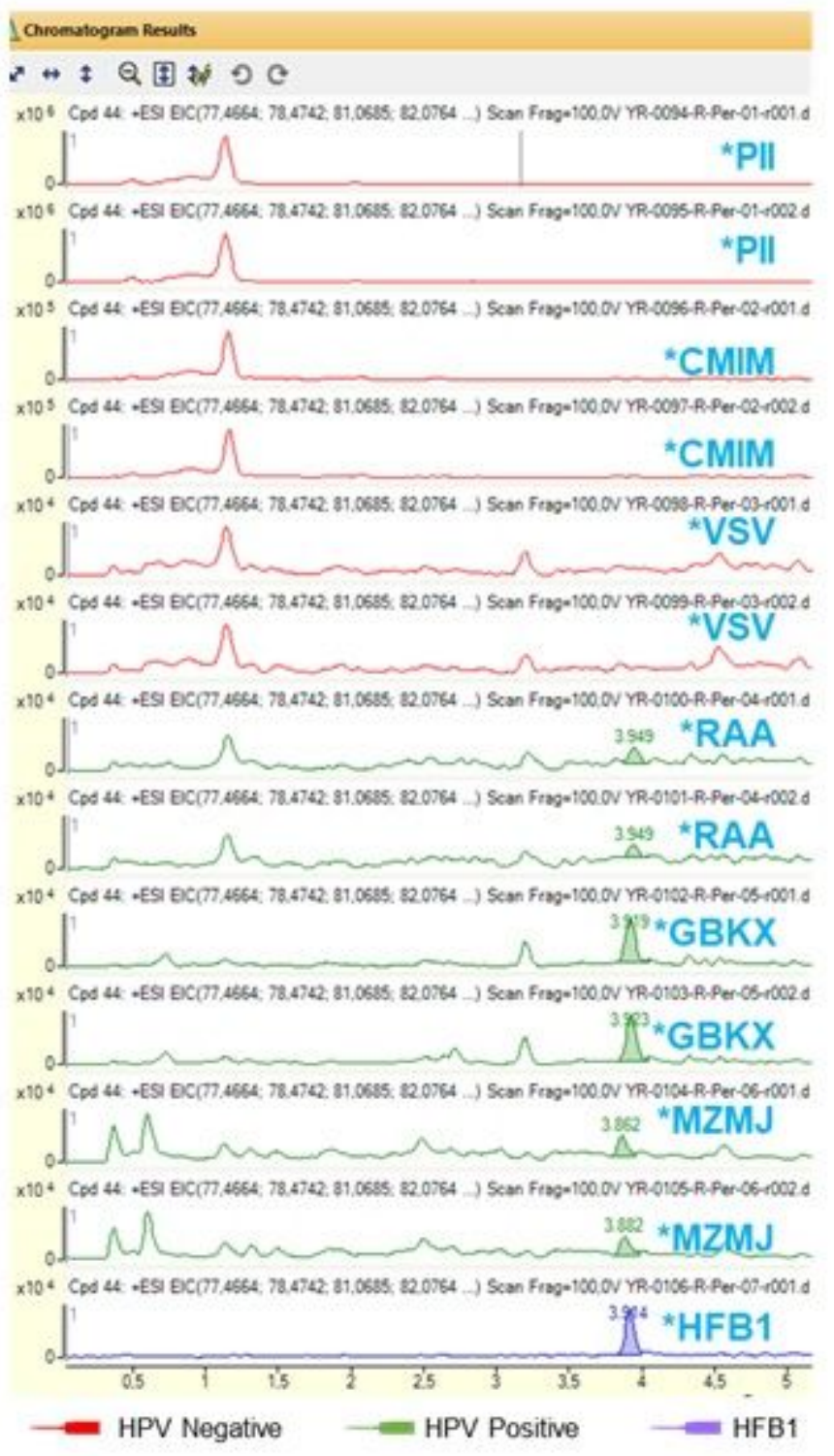

\section{Figure 2}

Chromatographic results for HFB1 (aminopentol). 


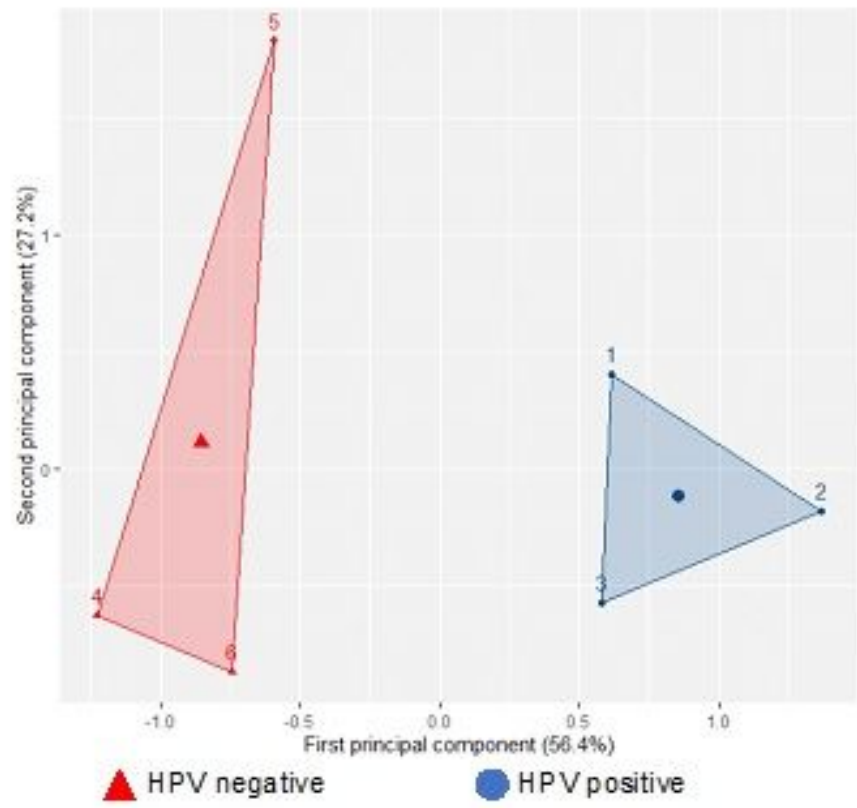

Figure 3

Biplot distribution of the subjects according to the two first principal components.

\section{Supplementary Files}

This is a list of supplementary files associated with this preprint. Click to download.

- SupplementaryMaterial.docx 